\title{
Determinants of Nutrition Care Practice by Midwives and Nurses in the Antenatal and Postnatal Care Settings: A Multi-Site Cross-Sectional Survey From Ghana
}

Global Pediatric Health

Volume 8: $1-12$

(C) The Author(s) 2021

Article reuse guidelines:

sagepub.com/journals-permissions

DOI: 10.1 I 77/2333794X21 1048382

journals.sagepub.com/home/gph

(S)SAGE

\author{
Jacqueline Kumbiley, $M S^{1}$, Anthony Amalba, $\mathbf{P h D}^{2,3}$, \\ Paul Armah Aryee, PhD', Simon Agongo Azure, MPhil2,3, \\ and Victor Mogre, $\mathrm{PhD}^{\prime}$ (D)
}

\begin{abstract}
Aim. Midwives and nurses are critical in nutrition care for pregnant women and lactating mothers. Ghanaian nurses and midwives' perception of the adequacy and their satisfaction with nutrition education received during training in school, level of nutrition care competencies, and nutrition practice behavior is unknown. We evaluated the adequacy of nutrition education received in nursing and midwifery school; nutrition care competencies; self-efficacy and the nutrition care practice of midwives and nurses. We also evaluated determinants of nutrition care practice during routine antenatal and postnatal care. Methods. Cross-sectional study conducted among midwives and nurses working at antenatal and postnatal clinics in Ghana. Data was collected using a self-administered questionnaire. Data analysis was done using descriptive statistics, correlation, and linear regression. Findings. Almost $90 \%(n=267)$ of the participants received nutrition education during training, $\mathbf{7 7 . 5 \%}$ were unsatisfied with the amount of time allocated for nutrition education and $40 \%$ felt inadequately prepared from school to provide nutrition care. Self-efficacy ranged from moderate to low. Determinants of nutrition care practice were age of respondents $(B=0.04 ; P=.002)$, nutrition-related knowledge $(B=0.05 ; P=.016)$, adequacy of nutrition education $(B=0.14 ; P=.006)$, nutrition training after school $(B=0.38 ; P=.010)$ and nutrition care self-efficacy $(B=0.03 ; P=.048)$. Conclusion. Notable knowledge gaps in basic nutrition, inadequate preparedness, and poor confidence to provide nutrition care was common. There is a need to improve the nutrition education experiences of midwives and nurses through curricula revision and refresher training courses.
\end{abstract}

\section{Keywords}

nutrition education, competency, self-efficacy, midwives, nurses, antenatal care and postnatal care

Received August 8, 2021. Accepted for publication September 6, 2021.

\section{Introduction}

A healthy childhood translates into a healthy adulthood. ${ }^{1}$ Healthy childhood can be achieved through healthy practices and care before, during, and after pregnancy. ${ }^{2}$ The proper development of the fetus and the health of the mother is dependent on healthy dietary practices during pregnancy. ${ }^{3}$ Unhealthy diet during pregnancy is reportedly associated with poor intrauterine growth restriction, low birthweight, gestational weight gain, and other adverse health outcomes in women and their newborn babies. ${ }^{4,5}$ Also, poor nutrition during pregnancy has been linked with a higher risk of developing chronic diseases in adulthood including cardiovascular diseases, diabetes, and poor bone health. ${ }^{6-8}$ The main causes of the higher rates of maternal and neonatal diseases and death in developing countries were due to poor nutrition. ${ }^{9}$

Ensuring good nutrition is essential to sustain the health of a woman during pregnancy, ensure normal healthy delivery, and lessen the risks of birth defects,

\footnotetext{
'University for Development Studies, Tamale, Ghana

${ }^{2}$ College of Health, Yamfo, Ghana

${ }^{3}$ Department of Population and Reproductive Health, University of

Port Harcourt, Nigeria

\section{Corresponding Author:}

Victor Mogre, Department of Health Professions Education and Innovative Learning, School of Medicine, University for Development Studies, PO Box TL 1883 Tamale, Ghana.

Email: vmogre@uds.edu.gh
} 
poor development of the fetus, and childhood health problems. ${ }^{10}$

The antenatal and postnatal care setting is an important medium to help pregnant women and mothers adopt healthy dietary habits. Thus, nutrition education given to mothers by healthcare providers during antenatal and postnatal care/visits can improve upon their nutrition and health status as well as that of their children. ${ }^{11,12}$ Nutrition education and counseling, largely referred to as nutrition care, forms part of antenatal and postnatal services. These services are mainly carried out by nurses and midwives, ${ }^{1,13-15}$ who are well placed to provide nutrition care during routine antenatal and postnatal care/visits and during their frequent contacts with pregnant women and lactating mothers. Their role to provide nutrition care is more manifest by the inadequate number of nutrition and dietetic professionals to offer nutrition care to pregnant women and lactating mothers during routine antenatal and postnatal care.

However, there is evidence ${ }^{16,17}$ showing that nurses and midwives who are in-charge of providing healthcare (including nutrition care) at postnatal and antenatal contact points barely provide nutrition care. A study conducted in Australia among midwives reported that midwives find it difficult to provide proper nutrition counseling to mothers. ${ }^{18}$ Two systematic reviews that included only articles from developed countries also reported that mothers received inadequate nutrition care during pregnancy. ${ }^{1,19}$ In Ghana, Nsiah-Asamoah ${ }^{20}$ investigated gaps in knowledge levels of health workers and reported that counseling caregivers on appropriate infant and young child feeding practices among these health workers was problematic. A number of barriers have been reported to militate the effective provision of nutrition care in the antenatal and postnatal care setting. These include inadequate nutrition training during school, lack of time, lack of resources, poor nutritionrelated knowledge, and inadequate confidence in nutrition care. ${ }^{1,18,19,21,22}$

Studies from developing countries investigating the provision of nutrition care during routine antenatal and postnatal care is limited. In our search for literature in this area we only came across information from

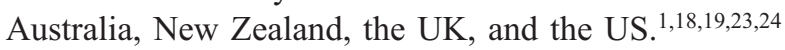
Only 1 study from Ghana investigated the nutritionrelated knowledge levels of health workers during routine growth monitoring and promotion but not during antennal care for pregnant women. ${ }^{20}$ The current study intends to fill these gaps in the literature. We investigated the provision of nutrition care and its determinants among midwives and nurses involved in routine antenatal and postnatal care in Ghana. Contextual findings are needed to inform interventions that aim to improve the provision of nutrition care by midwives and nurses during routine antenatal and postnatal care in developing countries. Evidence from this study will provide such contextual findings.

\section{Methods}

\section{Study Design and Setting}

Following an institution-based cross-sectional design, the study was conducted in the Sunyani Municipality of the Bono Region of Ghana. The Municipality is one of the 260 Metropolitan, Municipal and District Assemblies (MMDAs) in Ghana, and forms part of the 12 Municipalities and Districts in the Bono Region with the capital being Sunyani. It is located at the heart of the Bono Region lying between Latitudes $70^{\circ} 20^{\prime} \mathrm{N}$ and $70^{\circ} 05^{\prime} \mathrm{N}$ and Longitudes $20^{\circ} 30^{\prime} \mathrm{W}$ and $20^{\circ} 10^{\prime} \mathrm{W}$ and covers a total land area of $506.7 \mathrm{~km}^{2}$. The Municipality is advantaged to have the Regional Hospital cited in the capital city of Sunyani. The Municipal Health Directorate (MHD) is responsible for the health administration and coordination of activities of all health providers within the Municipality. It has 31 health facilities and 34 functional Community Based Health Planning and Services (CHPS) zones (3 compounds) in 6 sub-municipalities. Of these, 20 were selected including CHPS compounds for the study including the Bono Regional Hospital, Sunyani Municipal Hospital, Seventh-Day Adventist (SDA) Hospital, Opoku clinic, Abesim Health Center, Abesim Christian Eye clinic, Fiapre Health Center, Atronie Health Center, Watchman CHPS compound, Owusu Memorial Health Center, Yawhima Health Center, Yawsae Health Center, Antwikrom Health Center, Penkwase Clinic, Monica Maternity Home, Akyerensua CHPS compound, Abuom Rural Clinic, Abrikasu Health Center, and Ntotroso Health Center).

\section{Participants, Recruitment Procedures and Sample Size}

The study participants were health care providers (ie, nurses and midwives) working at the antenatal and postnatal contact points in the health facilities of the Sunyani Municipality. The health facilities run daily antennal and Child Welfare clinics (CWCs) for pregnant women and lactating mothers, scheduled to visit monthly to receive antenatal and postnatal care respectively. Participants eligible for the study were healthcare providers who have been working for at least 6 months at antenatal and postnatal contact points in the health facility. Those who had worked for less than 6 months were excluded from the study. A multi-stage sampling method was used for 
the selection of the health facilities and participants. Sampling was done by allocating numbers to 34 health facilities both private and public including CHPS compounds, and random selection was done to come out with a total of 20 facilities. With the use of a purposive sampling method eligible participants in the selected health facilities were invited to participate in the study. Sample size was estimated at 385 using the formula: $\left(z^{2} \times p(q)\right) / \mathrm{ME}^{2}{ }^{25}$ where $z=$ confidence level at $95 \%$ (standard value of 1.96), $p=$ estimated prevalence of nutrition care practice. Given that the prevalence of nutrition care practice has not been reported in Bono Region previously, $50 \%$ was assumed. $\mathrm{ME}^{2}=$ margin of error at $5 \%$.

\section{Data Collection Procedures}

Through the Regional Director of Health Services of the Bono Region, permission was sought from the management of the selected health facilities to gain access to them. All participants in the selected health facilities were approached by JK during routine antenatal and CWC days to invite them to participate in the study. The purpose of the study was introduced to the participants and all those who agreed to take part were taken through the consent procedures. Voluntary participation was encouraged and participants were assured of confidentiality of their responses. Those who consented were given copies of paper-based self-administered questionnaires to collect data. In order not to interfere with working hours of the participants, consented participants were allowed to fill the questionnaire in a secluded room in the facility whiles others were allowed to fill it at their own convenient time. Participants spent about 20 to 30 minutes filling the questionnaire. The data used in this study was collected as part of a thesis dissertation for JK. Ethical approval for all the data collection procedures and processes was granted by the Committee on Human Research, Publications and Ethics of the Kwame Nkrumah University of Science and Technology (Approval ID: CHRPE/AP/673/19). All procedures were performed in accordance with relevant guidelines.

\section{Data Collection Methods}

Data was collected using a self-administered questionnaire. For clarity and comprehensibility of the questions, a pre-test was conducted using 5 non-participants who were community health nurses and midwives. To determine content validity the items of the questionnaire were assessed by a team of nutritionists and behavioral scientists. The questionnaire was structured consisting of closed ended questions to assess adequacy of nutrition education during school, satisfaction with nutrition education, nutrition care competencies (ie, Knowledge, Attitude, Skills), self- efficacy in nutrition care, and nutrition care practice. Information on the general and background characteristics of the participants were also collected.

\section{Nutrition Education During School}

Items that assessed health care providers nutrition education involved a "Yes or No" response. Participants were asked to indicate how they received nutrition education when they were in school, whether they had it as "separate nutrition course," "lectures on selected topics," or "nutrition concepts integrated into course work."

(a) Amount of time spent on nutrition lectures in terms of credit hours (CH) per week in school: In order to assess the number of credit hours spent per week on nutrition lectures when they were in school, an open-ended question was used in which participants were asked the number of credit hours they spent on nutrition education while in school.

(b) Adequacy of nutrition education: To assess whether the nutrition education participants received in school was adequate, a 5-point Likert scale was used where participants were asked to indicate how adequately prepared their school has made them to provide nutrition care $(1=$ not at all adequate; 2 = somewhat adequate; $3=$ uncertain; $4=$ adequate; and $5=$ more than adequate).

(c) Nutrition training after school: To find out whether participants had nutrition training after school was assessed with the question "Apart from nursing/midwifery school, have you had any training on nutrition" Yes or No. To determine the kind of training received in nutrition if "Yes" 2 multiple questions and an open- ended question were used.

(d) Satisfaction with nutrition education: To determine participants level of satisfaction with some aspects of learning nutrition during training in school, 4 items were adapted from a previous study among Ghanaian medical doctors. ${ }^{22}$ Participants were asked to indicate how satisfied they were with some aspect of nutrition they learned during training in school using a 5-point Likert scale, rating them as follows $(1=$ not at all satisfied; $2=$ somewhat satisfied; $3=$ uncertain $4=$ satisfied; and 5=more than satisfied). The responses were used to generate a satisfaction score. 


\section{Nutrition Care Competencies}

The nutrition care competencies consisted of items that assessed health care providers knowledge, attitude, and skills regarding nutrition care.

(a) Knowledge: To test participants nutrition related knowledge level, questions were asked on maternal nutrition during and after pregnancy and nutrition relevant topics. The items of the knowledge scale of the questionnaire were adapted from previously published studies that assessed nutrition knowledge levels among nurses and midwives at antenatal care ${ }^{1,18,26}$ and others were author created. The knowledge scale for assessment consisted of 20 multiple-choice questions and 11 true or false questions. Each correctly answered question was scored 1 mark and summed to generate a total score that was computed out of $100 \%$.

(b) Attitude toward nutrition care: Regarding assessment of participants attitude toward nutrition care, an attitude scale was used where 7 items were presented as statements and defined on a 5-point Likert scale. Participants were required to determine the extent to which they agreed or disagreed to each of the statements by indicating $1=$ strongly disagree; 2 =disagree; $3=$ neutral; $4=$ agree; and $5=$ strongly agree. For instance, to what extent do you agree or disagree to the statement that "Nutrition counseling should be an integral part of routine care for all health care providers." Items of the attitude scale were adapted from previously published studies. ${ }^{27-29}$ Each answered question was scored a mark to generate a total score and computed out of $100 \%$. The items with the higher scores that were closer to $100 \%$ were denoted as positive attitude and lower scores being $50 \%$ and below were considered poor attitude.

(c) Nutrition care skills: The nutrition care skills scale had items that assessed participants degree of agreement or disagreement concerning selfreported skills they use to provide nutrition care during routine antenatal and postnatal care. The assessment scale consisted of 7 items in a form of statements defined on a 5-point Likert scale. Participants were required to determine the extent to which they agreed or disagreed to each of the statements by indicating $1=$ strongly disagree; 2 =disagree; 3 =neutral; $4=$ agree; and $5=$ strongly agree. For example, to what extent do you agree or disagree to the statement "the nutritional assessment of mothers is performed methodically and professionally." Similar to the scoring of the attitude scale, each item was scored to generate a total score for each participant.

(d) Nutrition care self-efficacy: The nutrition care self-efficacy was assessed with a scale comprising of 5 items relating to participants level of confidence in the delivery of nutrition care with the use of a 5-point Likert Scale ranging from 1 (not confident at all) to 5 (extremely confident). For example, "How confident or unconfident are you in discussing with lactating mothers the benefits of exclusive breastfeeding." Items were adapted from previous study among doctors. ${ }^{27}$ Each item was scored and a total score generated for each participant.

\section{Nutrition Care Practice}

Nutrition care practice was assessed with the question "What proportion of your clients/attendees do you actually provide nutrition care to." A 5-point Likert response was provided in which $1=$ less than $20 \% ; 2=20 \%-40 \%$; $3=41 \%-60 \% ; 4=61 \%-80 \%$, and $5=81 \%-100 \%$. The responses were scored to yield a nutrition care practice score. In addition, the following were also assessed using the questionnaire: kind of nutrition care participants provided, proportion of clients, participants believed will benefit from nutrition care, the time participants spent providing nutrition care such as counseling, and the number of times they referred mothers for specialist nutrition care in the last 6 months. These were assessed using "Yes" or "No" responses. Assessing whether participants referred mothers to dieticians/ nutritionist for specialist care was carried out using a "Yes or No" response. Open-ended questions were used to determine how many times they referred mothers in the past 6 months. Similarly, their reasons and kind of conditions for referral were also assessed.

\section{General and Background Characteristics}

A total of 12 questions were used to assess the general and background characteristics of the participants. These included their age, sex, type of practice, type of facility of practice, duration of practice, and level of education.

\section{Statistical Analysis}

The questionnaires were coded, entered, and analyzed using the International Business Machines CorporationsStatistical Package for Social Sciences (IBM SPSS) version 25.0 software. For easy description of the analyzed 
Table I. General and Background Characteristics $(n=300)$.

\begin{tabular}{lcc}
\hline Variables & Frequency & Percentage (\%) \\
\hline Gender & & \\
Male & 80 & 26.7 \\
Females & 220 & 73.3 \\
Level of education & 115 & 38.3 \\
Certificate & 149 & 49.7 \\
Diploma & 33 & 11.0 \\
Bachelor degree & 3 & 1.0 \\
Masters & & 38.0 \\
Type of practice & 114 & 62.0 \\
Midwife & 186 & \\
Community health nurse & & 22.0 \\
Type of facility & 66 & 20.3 \\
Regional hospital & 61 & 12.7 \\
District hospital & 38 & 21.3 \\
Public clinic & 64 & 23.3 \\
Private hospital & 70 & 0.3 \\
CHPS compound & 1 & \\
Other & & \\
\hline
\end{tabular}

data, descriptive statistics of mean, standard deviation, and frequencies were used. Pearson correlation was used to determine univariate associations among continuous variables (satisfaction with nutrition education, adequacy of nutrition, nutrition care competency, self-efficacy, and levels of nutrition care practice). One-way analysis of variance (ANOVA) and student $t$-test were used respectively to assess univariate associations among continuous variables (levels of nutrition care practice) and categorical variables (sex, having nutrition as a separate course, lectures on selected nutrition topics; ever had training on nutrition and nutrition concepts integrated into course work). To determine factors associated with nutrition care practice, a multiple linear regression was conducted. A $P$-value of $<.05$ was considered significant in all analyses.

\section{Results}

\section{General and Background Characteristics of the Participants}

Table 1 presents the general and background characteristics of the participants. Complete data was obtained from 300 participants out of an estimated sample size of 385; yielding a $78 \%$ response rate. As shown in Table 1, $73.3 \%(n=220)$ of the participants were females; $49.7 \%$ $(\mathrm{n}=149)$ had diploma certificate, and 62\% $(\mathrm{n}=186)$ were community health nurses. The participants mean (SD) age in years was $30.11 \pm 8.50$ and participants had been working foramean(SD)duration of $61.30 \pm 53.09$ months.

\section{Satisfaction Level and Adequacy of Nutrition Education Received During Training in School}

Almost all participants $(n=287,95.7 \%)$ received nutrition education during training in school in the form of lectures. Mean hours per week used for nutrition education during training was $3.31 \pm 1.66$ hours. More than half of the participants were either unsatisfied or uncertain with: the amount of time allocated for nutrition education during training in school (77.5\%); the integration of nutrition content into the curriculum (82.6\%); and how nutrition course content was presented (77.4\%). Participants had a mean (SD) satisfaction score of 10.01 (3.73). With a mean (SD) adequacy score of 3.31 (1.24), $61.4 \%(n=184)$ of the participants said they felt adequately prepared from school to provide nutrition care during routine antenatal and postnatal care.

\section{Participants' Nutrition-Related Knowledge, Attitude Toward Nutrition Care, and Self- Reported Nutrition Care Skills During Routine Antenatal and Postnatal Care}

Participants had total mean (SD) nutrition-related knowledge of $57.46(8.12) \%$. Regarding the different areas; participants had a mean (SD) knowledge score of 72.48 $(12.47) \%$ for maternal nutrition during pregnancy and $51.46(9.21) \%$ for infant and young child feeding practices. Over $80 \%$ of the participants knew that: balanced diet is important during pregnancy; energy requirements of pregnant women differ from non-pregnant women; the 
Table 2. Participants' Level of Self-Efficacy in Nutrition Care During Routine Antenatal and Postnatal Care.

\begin{tabular}{|c|c|c|c|c|}
\hline Level of self-efficacy items & $\begin{array}{l}\text { Not confident } \\
(\%)\end{array}$ & $\begin{array}{l}\text { Neither confident nor } \\
\text { unconfident }(\%)\end{array}$ & Confident (\%) & Mean \pm SD \\
\hline $\begin{array}{l}\text { Discuss with mothers on the importance and } \\
\text { challenges of breastfeeding }(n=297)\end{array}$ & $81(27.3)$ & $56(18.9)$ & $160(53.9)$ & $3.34 \pm 1.31$ \\
\hline $\begin{array}{l}\text { Using the food guide pyramid during counselling } \\
\text { to show examples of a serving size of certain } \\
\text { foods to mothers' }(n=296)\end{array}$ & $70(23.6)$ & $76(25.7)$ & $150(50.7)$ & $3.37 \pm 1.16$ \\
\hline $\begin{array}{l}\text { Discuss with pregnant and lactating mothers on } \\
\text { healthy eating habits }(n=295)\end{array}$ & $59(20)$ & $62(21.0)$ & $174(58.9)$ & $3.63 \pm 1.22$ \\
\hline $\begin{array}{l}\text { Discuss with mothers the content of calories } \\
\text { per gram of protein, carbohydrates, fat, and } \\
\text { their basic metabolic roles }(n=296)\end{array}$ & $93(31.4)$ & $77(26.0)$ & $126(42.6)$ & $3.13 \pm 1.25$ \\
\hline $\begin{array}{l}\text { Ability to provide nutrition education for } \\
\text { mothers recently diagnosed with HIV }(n=296)\end{array}$ & $|3|(44.3)$ & $32(10.8)$ & $133(44.9)$ & $3.09 \pm 1.42$ \\
\hline Total mean (SD) self-efficacy score & & & & $16.16 \pm 4.60$ \\
\hline
\end{tabular}

For easy rating of results, "Not very confident at all" and "not very confident" were combined to yield not confident whiles "confident" and "extremely" confident were combined to yield Confident.

nutrient needs for iron and folic acid is higher during pregnancy; nutrient deficiencies and being underweight can impact negatively on pregnancy outcomes; obesity during pregnancy can increase one's risk of several pregnancy complications. However, majority of the participants did not know: that iron is not a vitamin; the recommended daily intake of iron and folic acid for pregnant women; the recommended weight gain during pregnancy; that rich sources of iron and folic acid. Only $26 \%$ of the participants correctly answered that breastmilk only is adequate to meet the nutrient needs of infants aged less than 6 months; $47 \%$ said babies should be breastfed on demand; and $98 \%$ said babies younger 6 months could digest other foods. Less than $40 \%$ of the participants had knowledge on the benefits of exclusive breastfeeding to the baby and to the mother. Almost all participants knew the age of at which complementary breastfeeding should begin. Regarding foods that could added to enrich the diets of young children; $43 \%$ said animal sourced foods such as meat and eggs; $49 \%$ pulse and nuts, groundnut flours, and other legumes; $30.7 \%$ vitamin A rich fruits and vegetables such as carrots; $90.7 \%$ green leafy vegetables; and 13\% energy rich foods such as oil and butter. Regarding attitudes toward nutrition during pregnancy and lactation; participants had mean $\pm \mathrm{SD}$ attitude score of $23.00 \pm 4.56$. Majority $(81.7 \%, n=241)$ of the participants agreed that nutrition during pregnancy was very important; $72.4 \%$ said nutrition should be part of routine antenatal and postnatal care; and $63.9 \%$ believed that if mothers are counseled, they will adopt healthy dietary practices. However, $56.1 \%$ felt that midwives and nurses should also focus on nutrition support during routine antenatal and postnatal care. Also, $44.1 \%(n=130)$ of the participants agreed that dietitians rather than the nursing staff were solely responsible for nutritional support during routine antennal and postnatal care as $37.9 \%(n=112)$ disagreed with that statement. Participants had a mean (SD) nutrition care skill score of 18.58 (5.71). Less than half of the participants reportedly had skills to: perform nutrition assessment of mothers (41.7\%); effectively communicate nutrition information to mothers $(42.1 \%)$; accurately measure and record anthropometric measures of mothers (49.5\%); and identify mothers and children with nutrition deficiencies (46.9\%). Also, slightly above half of the participants reportedly said they had the skills: to perform nutritional screening $(50.9 \%)$ and to provide nutrition care to improve the dietary intake of pregnant women (54.1\%).

\section{Self-Efficacy in Nutrition Care}

As indicated in Table 2, 53.9\% of the participants said they were confident having discussions with mothers on the importance of exclusive breastfeeding for both mothers and babies; $58.9 \%(n=174)$ were confident in discussing healthy eating guidelines with mothers; and $42.6 \%$ in discussing with mothers the number of calories per gram of protein, carbohydrates and fat including their basic metabolic roles. Participants had a mean \pm SD self-efficacy score of $16.16 \pm 4.60$.

\section{Nutrition Care Practice During Routine Antenatal and Postnatal Care by the Participants}

Figure 1 shows the proportion of mothers, participants believed would benefit from nutrition care and the proportion of mothers that actually received nutrition care 
from participants (A) and the amount of time they spent doing so (B). Participants provided nutrition care at an average of 3.16(1.09) corresponding with $41 \%$ to $60 \%$ of the mothers they believed would benefit from nutrition care. There was a poor level of agreement between the proportion of mothers, participants believed would benefit from nutrition care and the proportion that actually received nutrition care (Kappa statistic $=.131$, $\mathrm{SE}=0.039, P<.001)$. With the time participants spent on nutrition care, $43.9 \%(\mathrm{n}=130)$ spent 21 to 30 minutes of their time providing nutrition care whiles only $3.4 \%$ $(n=10)$ spent above 40 minutes. Among the participants, $66.2 \%$ referred mothers to nutritionists/dieticians for specialized nutrition care and they did so for an average of 4.35 times in the last 6 months.

\section{Association Between Background Characteristics of Participants, Level of Satisfaction With Nutrition Education, Adequacy of Nutrition Education and Self- Efficacy With Levels of Nutrition Care Practice}

As shown in Table 3, adequacy of nutrition education $(r=.155 ; P<.001)$ and participants level of satisfaction with nutrition education $(r=.135 ; P=.020)$ correlated positively with levels of nutrition care practice. Attitudes correlated positively with nutrition care skills $(r=.523$; $P<.001)$, level of self-efficacy in nutrition care $(r=.368 ; P<.001)$, and nutrition knowledge $(r=.215$; $P<.001)$. Age correlated negatively $(r=-.120 ; P=.041)$ with nutrition-related knowledge of the participants but positively with levels of nutrition care practice $(r=.285$; $P<.001)$.

\section{Levels of Nutrition Care Practice Stratified by Sex and Format of Nutrition Education Received During School}

As shown in Table 4, level of nutrition care practice was significantly higher among participants who reportedly had: selected topics on nutrition ( 3.19 vs $2.50 ; P=.030$ ), nutrition concepts integrated into their course work (3.21 vs $2.57 ; P=.006$ ) and ever received training on nutrition after school (3.48 vs $2.89 ; P<.001)$.

\section{Multivariate Analysis of Factors Associated With Levels of Nutrition Care}

Table 5 presents a multiple linear regression of factors associated with levels of nutrition care practice. Having nutrition topics integrated into course work was

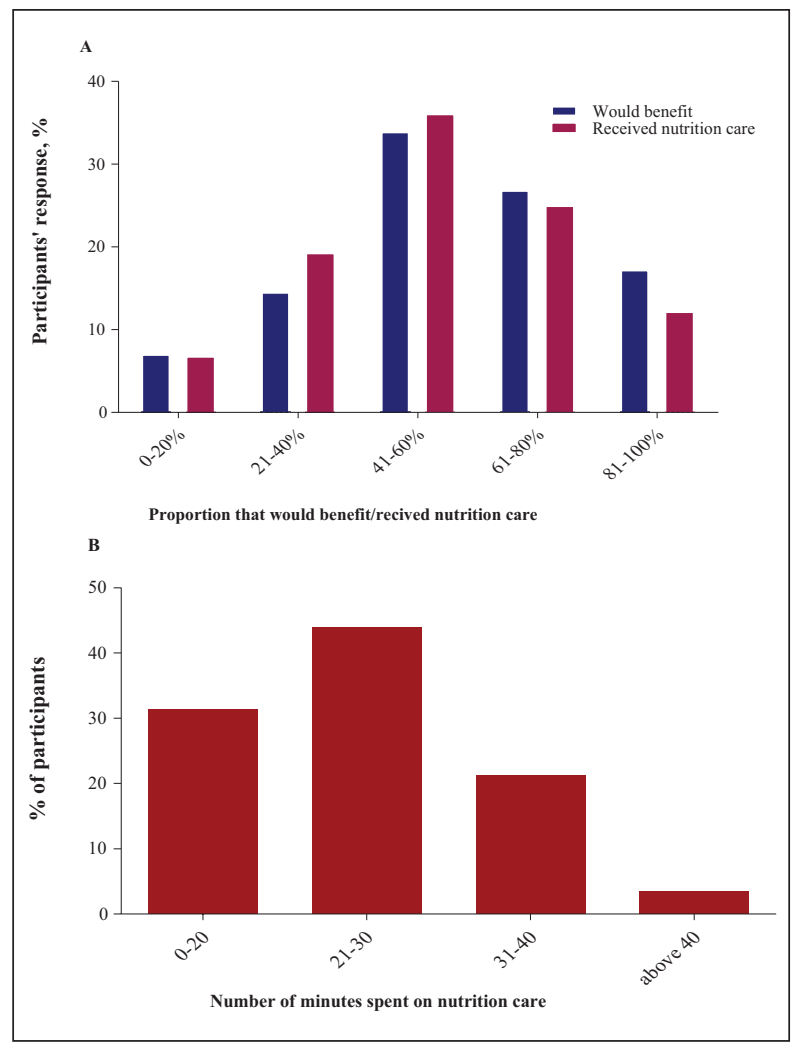

Figure I. Proportion of mothers' participants believed would benefit from nutrition care, the proportion of mothers that actually received nutrition care from participants $(A)$ and the amount of time they spent doing so (B) during routine antenatal and postnatal care.

significantly associated with increasing levels of nutrition care practice $(B=0.55, r=.147, P=.025)$, participants who reported to have received nutrition training after school recorded higher levels of nutrition care practice compared to those who said they did not receive training $(B=0.38, r=.169, P=.010)$. Increased level of self-efficacy in nutrition care was associated with increased levels of nutrition care practice $(B=0.03, r=.131 ; P=.048)$.

\section{Discussion}

An important finding of this study was that majority of the participants were unsatisfied with and inadequately prepared during their training in nursing and midwifery school to provide nutrition education in the antenatal and postnatal care setting. This finding is consistent with those reported in the international literature. ${ }^{1,18,19}$ The phenomenon of inadequate pre-service nutrition education is not peculiar to only nurses and midwives but other healthcare professionals such as doctors. Medical students and doctors from Ghana ${ }^{21,22}$ and other parts of the 
Table 3. Pearson Correlation of Participants Age, Working Experience, Adequacy, Satisfaction, and Self-Efficacy With Levels of Nutrition Care Practice.

\begin{tabular}{lccccccccc}
\hline Variable & WE & CH & ANE & SNE & ATN & NCS & SNC & NK & NCP \\
\hline Age & $.725^{* *}$ & $.136^{*}$ & -.002 & -.004 & .044 & -.114 & -.036 & $-.120^{*}$ & $.285^{* *}$ \\
Work experience (WE) & & -.006 & .077 & $.147^{*}$ & .049 & -.057 & .042 & -.065 & $.213^{* *}$ \\
Credit hours (CH) & & & $.175^{* *}$ & -.053 & .055 & -.006 & -.051 & .013 & .090 \\
Adequacy of nutrition education (ANE) & & & & $.246^{* *}$ & .003 & .113 & .061 & -.089 & $.155^{* *}$ \\
Satisfaction with nutrition education (SNE) & & & & & $.139 *$ & .082 & .059 & .069 & $.135^{*}$ \\
Attitude toward nutrition education (ANE) & & & & & & $.523 * *$ & $.368^{* *}$ & $.215^{* *}$ & .036 \\
Nutrition care skills (NCS) & & & & & & & $.609^{* *}$ & $.134^{*}$ & -.079 \\
Self-efficacy in nutrition care (SNC) & & & & & & & .071 & .058 \\
Nutrition knowledge (NK) & & & & & & & & $.134^{*}$ \\
\hline
\end{tabular}

Abbreviation: NCP, nutrition care practice.

*Correlation is significant at the .05 level (2-tailed).

**Correlation is significant at the .01 level (2-tailed).

Table 4. Mean Levels of Nutrition Care Practice Stratified by Gender and Format of Nutrition Education Received During School.

\begin{tabular}{|c|c|c|c|}
\hline Variable & Mean (SD) & $95 \% \mathrm{Cl}$ & $P$-value \\
\hline \multicolumn{4}{|c|}{ Gender $(n=296)$} \\
\hline Male & $3.19(1.05)$ & $2.82-3.35$ & \multirow[t]{2}{*}{.494} \\
\hline Female & $3.09(1.19)$ & $3.04-3.33$ & \\
\hline \multicolumn{4}{|c|}{ Nutrition as a separate course $(n=295)$} \\
\hline Yes & $3.17(1.10)$ & $3.03-3.30$ & \multirow[t]{2}{*}{.719} \\
\hline No & $3.09(1.00)$ & $2.73-3.45$ & \\
\hline \multicolumn{4}{|c|}{ Lectures on selected nutrition topics $(n=290)$} \\
\hline Yes & $3.19(1.07)$ & $3.06-3.32$ & \multirow[t]{2}{*}{.030} \\
\hline No & $2.50(1.17)$ & I.76-3.24 & \\
\hline \multicolumn{4}{|c|}{ Nutrition concepts integrated into the course work $(n=291)$} \\
\hline Yes & $3.21(1.07)$ & $3.08-3.33$ & \multirow[t]{2}{*}{.006} \\
\hline No & $2.57(1.04)$ & $2.12-3.01$ & \\
\hline \multicolumn{4}{|c|}{ Ever had training on nutrition after school $(n=289)$} \\
\hline Yes & $3.48(1.05)$ & $3.30-3.66$ & \multirow[t]{2}{*}{$<.001$} \\
\hline No & $2.89(1.02)$ & $2.73-3.05$ & \\
\hline
\end{tabular}

world have reported unsatisfaction with their nutrition education during medical school and feeling inadequately prepared to provide nutrition care in the general care setting. These findings demonstrate that healthcare professionals including nurses and midwives will benefit from further training in nutrition education. A number of factors have been identified for the inadequate nutrition education received during training in school including low priority for nutrition education; an already overcrowded curriculum; poor collaboration among dieticians/nutritionists and nursing and midwifery educators among others. ${ }^{1,18,19}$ Our finding in this study that participants who felt adequately prepared provided nutrition care to a higher number of mothers compared to those who reportedly felt inadequate to do so, demonstrate further that adequacy of nutrition education during training is crucial for improving upon nutrition care practices among health care providers. To buttress this point further we also found that participants who said nutrition concepts were integrated into their course work during nursing/midwifery training, were significantly more likely to report providing nutrition care to a higher proportion of mothers they believed would benefit from nutrition care. A possible explanation for our findings could be that improving adequacy of nutrition education may result in improved competency in nutrition care and self-efficacy that are required for an effective provision of nutrition care. One of the ways of making nutrition adequate in nursing and midwifery training is by integrating nutrition throughout the pre-service curriculum as this has been shown to be an effective way of improving nutrition education and subsequent nutrition care practice. . $^{1,30}$ 
Table 5. Multiple Linear Regression of Factors Associated With Levels of Nutrition Care Practice.

\begin{tabular}{|c|c|c|c|c|}
\hline Variable & $B$ & $95 \% \mathrm{Cl}$ & Partial correlation & $P$-value \\
\hline Male & -0.13 & -0.39 to 0.14 & -.061 & .356 \\
\hline Nutrition as a separate course & -0.04 & -0.50 to 0.42 & -.012 & .858 \\
\hline Had nutrition training after school & 0.38 & 0.09 to 0.67 & .169 & .010 \\
\hline Nutrition concepts integrated into course work & 0.55 & 0.07 to 1.02 & .147 & .025 \\
\hline Selected nutrition topics & 0.57 & -0.16 to 1.29 & .102 & .124 \\
\hline Age & 0.04 & 0.02 to 0.07 & .205 & .002 \\
\hline Number of credit hours in nutrition & 0.04 & -0.04 to 0.11 & .058 & .379 \\
\hline Adequacy of nutrition education & 0.14 & 0.04 to 0.25 & .180 & .006 \\
\hline Satisfaction with nutrition education & 0.03 & -0.01 to 0.06 & .088 & .183 \\
\hline Attitudes toward integration & -0.00 & -0.04 to 0.03 & -.003 & .960 \\
\hline Nutrition care skills & -0.03 & -0.06 to 0.00 & -.144 & .029 \\
\hline Self-efficacy in nutrition care & 0.03 & 0.00 to 0.07 & .131 & .048 \\
\hline Nutrition knowledge & 0.05 & 0.01 to 0.09 & .159 & .016 \\
\hline Work experience & -0.04 & -0.08 to 0.01 & -.101 & .125 \\
\hline
\end{tabular}

$F=4.55$, adjusted $R^{2}=.17$.

Participants had a mean knowledge score of $54.00 \%$, demonstrating suboptimal nutrition-related knowledge during pregnancy and lactation. A number of nutritionrelated knowledge deficits of the participants were identified. These were inadequate knowledge regarding food sources for micronutrients such as iron, folic acid, and vitamin $\mathrm{A}$; and the appropriate weight gain during pregnancy. Other areas of knowledge deficits were poor knowledge regarding feeding recommendations for infants younger than 6 months; benefits of exclusive breastfeeding and how to enrich foods for appropriate complementary feeding in young children. These findings are consistent with those of Arrish et $\mathrm{al}^{18}$ who reported inadequate knowledge of midwives regarding weight gain during pregnancy, recommended intake for iodine and food sources of iron. In addition, a systematic review $^{1}$ of studies from the international literature showed that midwives lacked basic knowledge on nutrient requirements, recommended weight gain, increased energy requirements, risk of iron-deficiency anemia, and folic acid requirements during pregnancy. Although these findings are concerning and may impact on the quality of nutrition care provided to mothers during routine antenatal and postnatal care, they provide important areas that could be mapped to inform pre-service curricula review and the content of in-service training programs to improve nutrition-related knowledge among nurses and midwives.

We also found that nutrition-related knowledge was associated with nutrition care practice as an increased nutrition-related knowledge corresponded significantly with increased nutrition care practice. Nutrition-related knowledge is an important first step required to help healthcare providers effectively communicate nutrition and dietary information to mothers that would enable them adopt healthy dietary practices. ${ }^{1,19}$ Consistent with our findings, Yalcin et $\mathrm{al}^{31}$ found that nurses who carried out nutrition assessment activities had a greater knowledge score than those who did not. Crogan and Evans ${ }^{32}$ also found a positive correlation between nutritional assessment activities and nutrition knowledge of nurses. However, it is important to note that knowledge is necessary but may not be sufficient to improve the nutrition care practice behavior. ${ }^{33}$ Other variables such as favorable attitudes, self-efficacy, and adequate skills may also have to be considered in designing interventions to improve nutrition care during routine antenatal and postnatal care. ${ }^{1,33}$

Generally, participants had a positive attitude toward the provision of nutrition care during routine antenatal and postnatal care. It is commendable that majority of the participants recognized that nutrition care should be part of routine care; that it is their responsibility to focus on providing nutrition support to mothers and they also believed that mothers will adopt healthy dietary practices if counseled to do so. These findings are consistent with those of previous findings conducted among midwives and nurses from Australia, New Zealand, the UK, and the US. ${ }^{1,18,19,23}$ The positive recognition of nutrition care suggests that participants will be willing to provide nutrition care to mothers if an enabling environment is provided and if they also feel adequately prepared to do so. It is however concerning that majority of participants did not think that nutrition counseling is an effective use of their professional time. This presents an interesting conundrum and suggests that participants have varying attitudes and inconsistent appreciation of the role of nutrition in maternal and child health outcomes and may negatively impact nutrition care during routine antenatal and postnatal care. 
Another important finding of this study was that participants generally lacked important skills to effectively provide nutrition care to mothers. We found that majority of the participants reportedly lacked skills to perform anthropometric measurements and interpret the results; provide nutrition counseling and communication; and perform nutrition screening to identify nutrition deficiencies and those at risk. This could be due to participants report of inadequate nutrition education received during school.

The self-efficacy levels of the participants were relatively average with most participants ranging from moderate to low level of confidence in discussing a number of nutrition issues. Participants felt less self-efficacious to provide nutrition care in a number of areas including encouraging mothers to exclusively breastfeed; counseling mothers to adopt healthy dietary habits; and the use of the food pyramid. These findings are comparable to those of previous findings and were not surprising given that participants reported inadequate nutrition education received during nursing and midwifery training., ${ }^{1,18}$ Nutrition care self-efficacy of participants affected the nutrition care practice as we found that increased selfefficacy was associated with increased nutrition care practice among participants. Comparable to our findings, Fisher et $\mathrm{al}^{30}$ reported that nurses' self-efficacy was related to knowledge and relevant clinical behavior which suggests a possible mediation effect of self-efficacy on the relationship between knowledge and behavior. Another study also reported a positive association between self-efficacy scores and the weight management practices of nurses. ${ }^{31}$

Another important finding of this study was that, although participants provided nutrition care to mothers during routine antenatal and postnatal care, the proportion of mothers that received the nutrition care did not correspond with the proportion of mothers they believed would benefit from nutrition care. This presupposes that there were a number of mothers that needed nutrition care but participants were unable to provide. This could be as a result of participants reportedly feeling less selfefficacious and inadequately prepared to provide nutrition care during routine antenatal and postnatal care.

In this study we did not find a positive association between nutrition care skills and participants nutrition care practice behavior. This finding is contrary to our assumption that health care providers who perceived themselves to have adequate skills should carry out nutrition care practices. A possible explanation for this could be due to the inadequacy of nutrition education reported by the participants. This probably resulted in poor nutrition-related knowledge and acquisition of nutrition care skills and subsequently inadequate provision of nutrition care. Another explanation could be that the nutrition education received during nursing training in schools focused largely on theory with less emphasis on the practical aspect of nutrition care. There is evidence that skill-based training or education for health professionals can help improve their self-efficacy and enhance their clinical performance including nutrition care practice. ${ }^{33}$

In-service training is another avenue to improve the nutrition care competencies and the dietary counseling skills of nurses and midwives to enable them effectively provide nutrition care. ${ }^{34,35} \mathrm{We}$ found that participants who reported receipt of nutrition training after school significantly reported providing nutrition care to a higher proportion of mothers, they believed would benefit from nutrition care compared to their counterparts who did not receive such training. These findings are similar to those reported from previous studies. ${ }^{1,18,19,36,37}$ These findings, thus demonstrate the positive effect of in-service nutrition training on health workers' nutrition knowledge, counseling skills, and management of nutrition related conditions and underscores the need for relevant authorities to institute inservice training programs in the form of continuous professional development programs (CPDs) on nutrition for health professionals to refresh their knowledge and to also build their confidence and the required attitude to provide nutrition care.

Another important finding of this study was that the age of participants was positively associated with nutrition care practice. This probably suggests that the older a person becomes, the more experienced he/she becomes regarding nutrition care, given that they were more likely to receive nutrition training at the work place, which has been shown in this study to be associated with nutrition care practice. Health care providers who are older may also feel more comfortable discussing health issues with their clients as they are perceived to be more knowledgeable and reliable source of nutritional information. As a result mothers are likely to presume them to be more experienced and provide accurate nutrition care, and will tend to rely on them for nutrition services as compared to the younger ones. ${ }^{38}$ Similar to the current study, an Australian study among practice nurses by Martin et $\mathrm{al}^{24}$ showed that majority of older practice nurses who were above the age of 50 years were perceived to be more experienced to provide nutrition counseling as compared to the younger practice nurses. Older healthcare providers could thus act as role models in the provision of nutrition care for the younger ones to emulate same. This is an important area that could be harnessed to improve the provision of nutrition care during routine antenatal and postnatal care.

This study has a number of strengths and limitations worth noting. It is one of its kind to investigate nutrition 
care practice during routine antenatal and postnatal care in a developing country like Ghana thus laying a foundation for future studies with similar interests. It has also provided evidence regarding the inadequacy and the unsatisfactory nutrition education experiences of nurses and midwives during training in school and brought to bear important points for curricula planning and change. Notwithstanding these strengths, the cross-sectional nature of the study made it difficult to establish causality. The generalizability of its findings is also limited due to the use of purposive sampling to select the study participants. The use of a questionnaire also increased the likelihood of social desirability as well as recall biases.

\section{Implications to Practice and Further Research}

The findings of this study have increased our understanding of the situation of nutrition education in nursing and midwifery schools in Ghana and that the nutrition care competencies of nurses and midwives is inadequate due to inadequate nutrition education received during training. A suggestion for further research is to find out whether nutrition information given to mothers by health care providers during their routine antenatal and postnatal services influence their health and nutritional status as well as that of their children. There is a need for future studies to explore further to identify the reasons for the inadequate nutrition education received by nurses and midwives during training in school. In addition, there is also the need for future studies to unravel the reasons for the negative association between self-reported skills and the level of provision of nutrition care in the current study. Also, future studies should investigate whether a nutrition training program will improve the nutrition practice behavior of nurses and midwives during routine antenatal and postnatal care.

\section{Conclusion}

Nutrition education received during nursing or midwifery training was reportedly unsatisfactory and inadequate, resulting in participants reporting sub-optimal nutrition-related knowledge, feeling ill prepared, and less confident in providing nutrition care. Participants also had inadequate key nutrition care skills needed to effectively provide nutrition care in antenatal and postnatal care settings. Despite participants recognizing nutrition care to be important and perceiving it to be one of their responsibilities they could not provide nutrition care to meet the needs of mothers during routine antenatal and postnatal care. As an initial step toward improving nutrition education and subsequently improving the provision of nutrition care by nurses and midwives, more research focusing on exploring barriers and enablers of nutrition education are needed. This would enable the identification and subsequent implementation of appropriate educational interventions to improve nutrition care competencies, self-efficacy, and nutrition care practice in the antenatal and postnatal care settings.

\section{Authors' Note}

Prior publication: This is manuscript is submitted as a Preprint to Research Square. Preprint DOI: 10.21203/rs.3.rs-376448/v1.

\section{Acknowledgments}

Authors wish to thank the administration of the health sector and facilities of the Bono Region and Municipality for granting them permission to the health facilities.

\section{Author Contributions}

JK conceived the study, designed it and collected data; VM designed the study, interpreted the data, performed data analysis, drafted the manuscript; SAA partly performed data analysis; AA and PA jointly undertook critical revision of the manuscript. All authors approved the manuscript for publication.

\section{Declaration of Conflicting Interests}

The author(s) declared no potential conflicts of interest with respect to the research, authorship, and/or publication of this article.

\section{Funding}

The author(s) received no financial support for the research, authorship, and/or publication of this article.

\section{ORCID iD}

Victor Mogre (iD https://orcid.org/0000-0003-0230-5783

\section{References}

1. Arrish J, Yeatman H, Williamson M. Midwives and nutrition education during pregnancy: a literature review. Women Birth. 2014;27(1):2-8.

2. Pasinlioglu T. Health education for pregnant women: the role of background characteristics. Patient Educ Couns. 2004;53(1):101-106.

3. Saravanan P, Yajnik CS. Role of maternal vitamin B12 on the metabolic health of the offspring: a contributor to the diabetes epidemic? Br J Diab Vasc Dis. 2010;10(3):109-114.

4. Landis SH, Lokomba V, Ananth CV, et al. Impact of maternal malaria and under-nutrition on intrauterine growth restriction: a prospective ultrasound study in Democratic Republic of Congo. Epidemiol Infect. 2009;137(2):294-304. 
5. Tovar A, Must A, Bermudez OI, Hyatt RR, Chasan-Taber L. The impact of gestational weight gain and diet on abnormal glucose tolerance during pregnancy in Hispanic women. Matern Child Health J. 2009;13(4):520-530.

6. Martyn CN. Fetal and infant origins of cardiovascular disease. Midwifery. 1994;10(2):61-66.

7. Barker DJ, Hales CN, Fall CH, Osmond C, Phipps K, Clark PM. Type 2 (non-insulin-dependent) diabetes mellitus, hypertension and hyperlipidaemia (syndrome $\mathrm{X}$ ): relation to reduced fetal growth. Diabetologia. 1993;36(1):62-67.

8. Jones G, Riley MD, Dwyer T. Maternal diet during pregnancy is associated with bone mineral density in children: a longitudinal study. Eur J Clin Nutr. 2000;54(10):749-756.

9. Black RE, Allen LH, Bhutta ZA, et al. Maternal and child undernutrition: global and regional exposures and health consequences. Lancet. 2008;371(9608):243-260.

10. Kaiser L, Allen LH; American Dietetic Association. Position of the American dietetic association: nutrition and lifestyle for a healthy pregnancy outcome. J Am Diet Assoc. 2008;108(3):553-561.

11. Everette M. A review of nutrition education: before, between and beyond pregnancy. Curr Womens Health Rev. 2009;5(4):193-200.

12. Garg A, Kashyap S. Effect of counseling on nutritional status during pregnancy. Indian J Pediatr. 2006;73(8):687-692.

13. Szwajcer EM, Hiddink GJ, Koelen MA, van Woerkum CM. Written nutrition communication in midwifery practice: what purpose does it serve? Midwifery. 2009;25(5):509-517.

14. Lincetto $\mathrm{O}$, Mothebesoane-Anoh S, Gomez P, Munjanja S. Antenatal care. In: World Health Organization, eds. Opportunities for Africa's Newborns: Practical Data, Policy Programmatic Support for Newborn Care in Africa, WHO; 2006:55-62.

15. Warren C, Daly P, Toure L, Mongi P. Postnatal care. In: World Health Organization, eds. Opportunities for Africa Newborn: Practical Data, Policy and Programmatic Support for Newborn Care in Africa. WHO; 2005;79-90.

16. Lee DJ, Haynes CL, Garrod D. Exploring the midwife's role in health promotion practice. Br J Midwifery. 2012;20(3):178-186.

17. Wennberg AL, Hamberg K, Hörnsten A. Midwives' strategies in challenging dietary and weight counselling situations. Sex Reprod Healthc. 2014;5(3):107-112.

18. Arrish J, Yeatman H, Williamson M. Australian midwives and provision of nutrition education during pregnancy: a cross sectional survey of nutrition knowledge, attitudes, and confidence. Women Birth. 2016;29(5):455-464.

19. Lucas C, Charlton KE, Yeatman H. Nutrition advice during pregnancy: do women receive it and can health professionals provide it? Matern Child Health J. 2014;18(10):2465-2478.

20. Nsiah-Asamoah C. Gaps in knowledge levels of health workers on recommended child feeding practices and growth monitoring and promotion actions. Pediatr Health Res. 2018;3(2):8.

21. Mogre V, Stevens FCJ, Aryee PA, Amalba A, Scherpbier AJJA. Future doctors' perspectives on health professionals' responsibility regarding nutrition care and why doctors should learn about nutrition: a qualitative study. Educ Health. 2019;32(2):91-94.
22. Mogre V, Stevens FCJ, Aryee PA, MatorwmasenAkkermans FL, Abubakari B, Scherpbier AJJA. Nutrition care practices, barriers, competencies and education in nutrition: a survey among Ghanaian medical doctors. Med Sci Educ. 2018;28(4):815-824.

23. Elias S, Green T. Nutrition knowledge and attitudes of New Zealand registered midwives. Nutr Diet. 2007;64(4): 290-294.

24. Martin L, Leveritt MD, Desbrow B, Ball LE. The selfperceived knowledge, skills and attitudes of Australian practice nurses in providing nutrition care to patients with chronic disease. Fam Pract. 2014;31(2):201-208.

25. Cochran WG. Sampling Techniques. Wiley; 1977.

26. Lim Z, Wong J, Lim P, Soon L. Knowledge of nutrition during pregnancy and associated factors among antenatal mothers. Int J Public Health Clin Sci. 2018;5(1): 117-128.

27. Mogre V, Aryee PA, Stevens FCJ, Scherpbier AJJA. Future doctors' nutrition-related knowledge, attitudes and self-efficacy regarding nutrition care in the general practice setting: a cross-sectional survey. Med Sci Educ. 2017;27(3):481-488.

28. Keaver L, O’Meara C, Mukhtar M, McHugh C. Providing nutrition care to patients with chronic disease: an Irish teaching hospital healthcare professional study. J Biomed Educ. 2018;2018:1-7.

29. Moses H. Nutrition Knowledge, Attitude and Practice of Professional Health Workers in Morogoro Urban District. Sokoine University of Agriculture; 2010.

30. Fisher LK. School nurses' perceptions of self-efficacy in providing diabetes care. J Sch Nurs. 2006;22:223-228.

31. Yalcin N, Cihan A, Gundogdu H, Ocakci AF. Nutrition knowledge level of nurses. Health Sci J. 2013;7(1):99.

32. Crogan NL, Evans BC. Nutrition assessment: experience is not a predictor of knowledge. J Contin Educ Nurs. 2001;32(5):219-222.

33. Mogre V, Scherpbier AJ, Stevens F, Aryee P, Cherry MG, Dornan T. Realist synthesis of educational interventions to improve nutrition care competencies and delivery by doctors and other healthcare professionals. BMJ Open. 2016;6(10):e010084.

34. Fletcher A, Carey E. Knowledge, attitudes and practices in the provision of nutritional care. Br J Nurs. 2011;20:615-616, 618-620.

35. Jefferies D, Johnson M, Ravens J. Nurturing and nourishing: the nurses' role in nutritional care. J Clin Nurs. 2011;20:317-330.

36. Sunguya BF, Poudel KC, Mlunde LB, et al. Effectiveness of nutrition training of health workers toward improving caregivers' feeding practices for children aged six months to two years: a systematic review. Nutr J. 2013;12(1):66.

37. Barrowclough D, Ford F. A nutrition open- learning pack for practising midwives. Nutr Food Sci. 2001;31(1):6-12.

38. Munuo AE, Mugendi BW, Kisanga OA, Otieno GO. Nutrition knowledge, attitudes and practices among healthcare workers in management of chronic kidney diseases in selected hospitals in Dar es Salaam, Tanzania; a cross-sectional study. BMC Nutr. 2016;2(1):1-7. 